\title{
Cytotoxicity and Cellular Uptake of 5-Fluorouracil Loaded Methylcellulose Nanohydrogel for Treatment of Oral Cancer
}

\author{
Gayatri Patel ${ }^{1 *} * \mathbb{C}$, Chintan Dalwadi ${ }^{1}$ \\ 1 Charotar University of Science and Technology, Ramanbhai Patel College of Pharmacy, Department of Pharmaceutics \& \\ Pharmaceutical Technology, CHARUSAT Campus Changa-388 421, Gujarat, India \\ * Correspondence: gayatripatel26@gmail.com;
}

Scopus Author ID 35500684500

Received: 17.08.2020; Revised: 26.09.2020; Accepted: 29.09.2020; Published: 3.10.2020

\begin{abstract}
The objective of the study was to investigate the cytotoxicity and cellular uptake of prepared 5-Fluorouracil (5-FU) nano hydrogel formulation using KB oral cancer cell line and VERO fibroblast cell line. The biodegradable thermoresponsive modified methylcellulose (MMC) polymer was used for the preparation of nano hydrogel, whereas it shows a sol-gel phase transition at $36^{\circ} \mathrm{C}$ to $40^{\circ} \mathrm{C}$. The physical crosslinking method was used, followed by probe sonication for the preparation of 5-FU loaded MMC nano hydrogel. The mechanism of crosslinking was studied by FT-IR and SEM. These experimental techniques explained physical crosslinking viz. H-bonding phenomenon with the interconnected porous structure of nanoscale pore size. Cytotoxicity assay in the concentration range from $1 \mu \mathrm{g} / \mathrm{ml}$ to $2000 \mu \mathrm{g} / \mathrm{ml}$ shows an IC50 value at around $250 \mu \mathrm{g} / \mathrm{ml}$. This nano hydrogel shows concentration-dependent toxicity to the cancer cells while they were less toxic to normal fibroblast VERO cells. Cellular uptake was confirmed by the green fluorescence inside cells by Rhodamine-B conjugation using a fluorescent microscope. These results depict that 5-FU loaded thermosensitive nano hydrogel may be a promising candidate for intratumoral targeted drug delivery to cancer cells.
\end{abstract}

Keywords: crosslinking; cytotoxicity; swelling; thermosensitive; tumor; viscosity

(C) 2020 by the authors. This article is an open-access article distributed under the terms and conditions of the Creative Commons Attribution (CC BY) license (https://creativecommons.org/licenses/by/4.0/).

\section{Introduction}

Head and neck cancers are the 6th most common cancer worldwide, with more than 600,000 new cases reported annually [1]. Studies have estimated the global incidence of all head and neck cancers to be between 400,000 and 600,000 new cases per year and the mortality rate to between 223,000 and 300,000 deaths per year. In the United States, more than 45,000 new cases of head and neck cancers were diagnosed each year, accounting for more than $3 \%$ of all incidence malignancy [2]. Overall, $57.5 \%$ of global head and neck cancers occur in Asia, especially in India, for both sexes. Head and neck cancers in India accounted for $30 \%$ of all cancers in males and 11 to $16 \%$ in females [3]. It is emerging as major public health problems, which are lifestyle-related, have a lengthy latent period, and need dedicated infrastructure and human resources for treatment. Over 200,000 cases of head and neck cancers occur each year in India. Among them, over 80000 oral cancers are diagnosed every year in the country [4]. Squamous cell carcinomas amount to more than $90 \%$ of malignant tumors of the oral cavity and oropharynx. In some regions, particularly in the Indian subcontinent, oral cancer is among the most frequent malignancies, largely due to tobacco chewing [5]. 
The best strategy for fighting cancer is prevention - i.e., making changes in lifestyles to reduce cancer risk. Even though we used all the techniques for preventing cancers, one out of four cancers would still occur. Because of this, therapies that target malignancies after they have developed will continue to be important for some time to come [6]. The most commonly used treatment modalities for cancer include some combination of surgery, radiation therapy, and chemotherapy. The excision of the tumor is the most frequently employed form of tumor therapy. More patients are cured of cancer with surgery than any other treatment method. But it is difficult to achieve complete removal of the tumor through surgery. In recent years, combining it with other treatment modalities such as chemotherapy and radiation therapy has enhanced the effectiveness of surgery.

Conventional systemic chemotherapy has been shown to produce side effects. Most of the drug content is released soon after administration causing drug levels in the body to rise rapidly, leading to unacceptable side effects at the peaks and inadequate therapy at the troughs. Cytotoxic drugs are extensively transported to the whole body; therefore, only a small fraction of the drugs reach the tumor site, and other healthy organs or tissues can be affected or damaged by the nonspecific action of the cytotoxic agents. Due to the short period of actions, repeated injections are often required, which can lead to exacerbation of side effects and inconvenience $[7,8]$.

To overcome the limitations of surgery and radiation therapy and conventional systemic controlled, targeting, or localized chemotherapy is almost always employed for the treatment of solid tumors [9]. National Cancer Institute has also developed a goal of localizing cancer therapy by using nanoparticles for localized chemotherapy, radiation, immunotherapy, hyperthermia delivery, or a combination of these therapies [10]. A few products based on this approach have been approved by the FDA for cancer treatment such as Lupron $\operatorname{depot}^{\circledR,}$, which contains sterile lyophilized microspheres when mixed with diluent, becomes a suspension intended as a monthly injection [11]. There are many products of nano hydrogel, which is under clinical trials for localizing cancer therapy [12]. Targeted drug delivery has the advantages of low toxicity, few side effects, and enhanced therapeutic efficacy of cancer chemotherapy.

Among the many anticancer drugs used in clinics, 5- fluorouracil (5-Fu), a hydrophilic anticancer drug, is extensively employed in systemic chemotherapy of solid tumors such as head and neck cancer, breast, colorectal, and brain cancer. It is a pyrimidine analog that inhibits the biosynthesis of deoxyribonucleotides for DNA replication by inhibiting thymidylate synthase activity, leading to thymidine depletion, incorporation of deoxyuridine triphosphate into DNA, and cell death [13,14]. However, 5-FU has limitations such as short biological halflife due to rapid metabolism, incomplete and non-uniform oral absorption, variable bioavailability, and non-selective action against healthy cells. To prolong the circulation time of 5-FU and to increase its efficacy, its delivery has to be modified. Therapeutic concentrations of 5-FU could be maintained by such targeted delivery that holds the therapeutic concentration of 5-FU and reducing systemic drug levels $[15,16]$.

In recent years, an injectable in-situ forming hydrogel has attracted considerable attention because it can achieve site specific drug delivery, prolonged action periods, and improved patient compliance [17]. In the use of hydrogels, obstacles are their macroscopic dimensions and quick elution of drugs from the swollen hydrogel matrix. These challenges can be addressed through the use of nanohydrogels. Nanohydrogels simultaneously possess features and characteristics of hydrogels and nanosystems. Therefore, they benefit from the hydrophilicity, flexibility, versatility, high water absorptivity, and biocompatibility, along with 
all the advantages of nanoparticles $[18,19]$. Nanoscale drug delivery vehicles formulated from biodegradable and biocompatible, thermo-responsive polymers constitute an evolving approach to drug delivery and tumor targeting.

Hydrogels or nanohydrogels can be implemented in a number of ways for the treatment of cancer. It includes, implanted near or within a tumor, placed at a tumor site post-resection, in-situ forming hydrogel or nano hydrogel injected intratumorally. A few products based on hydrogel have also been approved by the FDA for cancer treatment [20,17].

In this paper, we are describing such a targeted drug delivery vehicle with the intention of delivering 5-FU only at the tumor site by exploiting the LCST nature of the methylcellulose (MC) carrier. Here, we have prepared a nano hydrogel using modified methylcellulose (MMC) hydrogel polymer and characterize the gelling phenomenon, morphology, and stability of the formulation as well as proves the cytotoxicity and in-vitro cellular uptake using KB and VERO cell line.

\section{Materials and Methods}

\subsection{Materials.}

Methylcellulose (MC), with the brand name of METHOCEL, was obtained as a gift sample from Colorcon Asia Pvt. Ltd. Goa, India. Specifications of MC as per the manufacturer show that viscosity of $2 \% \mathrm{w} / \mathrm{v}$ solution at $20^{\circ} \mathrm{C}$ is about $400 \mathrm{cP}$, average molecular weight, polydispersity index, and average degree of methyl substitution reported being 130,000, 1.8, and 2.1 respectively. 5-FU was purchased from Zydus Cadila Healthcare Ltd., Ahmedabad. Different salts of analytical grade were purchased from Merck Limited, Mumbai, India. Deionized water was used solely for the experiment. All other reagents were of suitable analytical grade.

\subsection{Materials.}

2.2.1. Formulation development.

The Modified methylcellulose (MMC) hydrogel was prepared by the physical crosslinking method and then converted to nano hydrogel using probe sonication as a method reported by Giorgia Arrigo et al. [21]. MC solution was prepared by dispersing the accurately weighed amount of MC powder in de-ionized water at room temperature, followed by keeping it in a refrigerator for at least $12 \mathrm{hr}$ to obtain a clear solution. Then, accurately weighed $3 \% \mathrm{w} / \mathrm{v}$ Tri-sodium citrate (TSC) was added and dissolved in the prepared polymer solution using a magnetic stirrer. This solution was kept in a refrigerator at least $12 \mathrm{hr}$ to obtain a clear solution. 5-FU was added and dissolved to the prepared hydrogel. Nanohydrogel was prepared by sonication of the 5-FU loaded hydrogel solution using tip probe-sonicator settings of in pulse for 60 seconds at $20 \mathrm{kHz}$. This nano hydrogel solution was freeze-dried using lyophilizer (Advantage, SP Scientific, USA) at $-70^{\circ} \mathrm{C}$ in the primary freezing step for $8 \mathrm{hr}$ and then at $30^{\circ} \mathrm{C}$ in the primary drying phase for $22 \mathrm{hr}$. 
2.2.2. Evalution and characterization.

\subsubsection{Fourier transmission infrared spectroscopy (FT-IR) analysis.}

FT-IR spectrum of the freeze-dried nano hydrogel solution was recorded to check the gel formation phenomenon viz. Physical crosslinking of the MC and TSC using Nicolet FT-IR 5700 Spectrophotometer (Thermo Fischer Scientific, USA) at ambient condition. MC, TSC, and dried nano hydrogel samples were mixed with $\mathrm{KBr}$ and pressed to make a pellet and then analyze from 500 to $4000 \mathrm{~cm}^{-1}$ [22].

\subsubsection{Gelation time and gelling temperature.}

Gelation was assessed using the test tube tilting method (TTM). An aliquot of a two ml sample was filled in small glass tubes and equilibrated in a temperature-controlled water bath. Gelation was checked by inverting the tubes and observed if the gel flowed or not. The time and temperature at which the gel did not flow were recorded as the gelation time and gelation temperature.

\subsubsection{Rheological measurements.}

Rheological evaluations were carried out to check the flow behavior. It includes viscosity, gel strength, and syringe ability of the prepared 5-FU loaded MMC nano hydrogel solution. The viscosity of the sample with respect to shear rate and shear stress at room temperature $\left(25^{\circ} \mathrm{C} \pm 3^{\circ} \mathrm{C}\right)$ was assessed using a Rheometer (MCR301-Rheolab QC, Anton par, Austria). An aliquot of $4 \mathrm{ml}$ of solution was taken, and DG-26.7 spindle. Gel strength was measured by using Brookfield's Texture Analyzer - QTS 25 (Massachusetts, USA) by probe type Acrylic-38. Syringebility of the nano hydrogel solution was measured using the same instrument by taking WFI as a reference using a one ml 24G prefilled syringe [23].

\subsubsection{Swelling study.}

The swelling study was performed by immersing already weighed dried of the 5-FU loaded MMC nano hydrogel in phosphate buffer solution (PBS) pH 7.4 in preweighed containers for known intervals of time. The sample was placed in PBS and equilibrated to $37^{\circ} \mathrm{C}$ until the weight of the nanohydrogels did not further increase. The medium was carefully withdrawn at intervals, and wet weight was measured until equilibrium in weight was attained. The percentage swelling ratio was calculated using Equation 1 [24].

$$
\text { Percentage Swelling Ratio }=\frac{\text { Final Weight-Initial Weight }}{\text { Initial Weight }} \times 100 \quad \text { (Equation 1) }
$$

\subsubsection{In-vitro drug release.}

Drug release study of the market formulation and 5-FU nano hydrogel formulation was performed by dialysis tube (molecular weight cut-off $12 \mathrm{kDa}, 2.4 \mathrm{~nm}$ pore size, Himedia, India) method described by Yajing Wang et al., [25]. One ml of drug-loaded nano hydrogel solution containing $10 \mathrm{mg}$ 5-FU was filled into a pre-swelled activated dialysis bag. Drug release was measured by immersing the filled dialysis tube in $20 \mathrm{ml}$ of donor media PBS pH 7.4 on the magnetic stirrer at $37 \pm 5^{\circ} \mathrm{C}$ temperature with gentle agitation [26]. Aliquots of a predetermined quantity of three $\mathrm{ml}$ were collected manually at a definite time interval, and an equal volume 
of fresh PBS was immediately added to the incubation medium. Drug concentration was analyzed by U.V.Visible Spectrophotometer at $266 \mathrm{~nm}$ using the calibration curve equation. The concentration of drug released from nano hydrogel was expressed as a percentage of drug in nano hydrogel and plotted as a function of time. The release data was fitted in various kinetic models like zero order, first order, Higuchi, Korsmeyer-Peppas model [27].

\subsubsection{Morphology study.}

Scanning Electron Micrographs (SEM) (JSM-5600LV instrument, Japan) was used to observe the interior pores morphology of the nanohydrogels. Nanohydrogels swollen to equilibrium at room temperature were frozen in liquid nitrogen and snapped immediately, and then freeze-dried in a lyophilizer system to completely remove the water. After drying, the microstructures and morphologies of the cross-sections of the nanohydrogels were then visualized by an SEM [28].

\subsubsection{Cell culture.}

Cell culture methods were performed to evaluate the cytotoxicity of prepared nano hydrogel towards oral cancer KB cell line and normal fibroblast VERO cell line. This two KB and VERO cell lines were obtained from the National Repository of Animal Cell Culture, National Centre for Cell Sciences (NCCS), Pune, India. Both cell lines were cultured in $25 \mathrm{~cm}^{2}$ tissue culture flasks and maintained in Minimum Essential Medium (MEM) supplemented with $10 \%$ fetal bovine serum (FBS) and $1 \%$ penicillin, streptomycin, and amphotericin B (PSA) antibiotic mixture. The cells were incubated in an incubator with $5 \% \mathrm{CO}_{2}$. After reaching confluency, the cells were detached from the flask using trypsin-EDTA solution, and the cell suspension was centrifuged at Relative Centrifugal Force of 200 grams to 700 grams for 5 min to get a cell pellet. Cells were resuspended in the fresh medium as per the required concentration for further studies [15].

\subsubsection{Cytotoxicity assay.}

The viability of cancer cells treated with a 5-FU drug solution, 5-FU nano hydrogel, and MC polymer solution was studied by indirect method MTT colorimetric assay. For MTT cell viability assay KB and VERO cells were taken on a 96-well plate with a density of 10,000 cells per well and incubated for $24 \mathrm{hr}$ to allow cell attachment. Following attachment, the medium was substituted with medium containing prepared drug-loaded nano hydrogel sample at concentrations ranging from $1 \mu \mathrm{g} / \mathrm{ml}$ to $2000 \mu \mathrm{g} / \mathrm{ml}$ and incubated for $24 \mathrm{hr}$. After treatment, the cells were washed with PBS and incubated with $20 \mu \mathrm{l}$ per well MTT solution $(5 \mathrm{mg} / \mathrm{ml})$ for $3 \mathrm{hr}$ in the dark. Then, MTT was removed, and MTT formazan crystals were dissolved in 100 $\mu l$ Dimethyl Sulfoxide (DMSO). The results were measured by the ELISA plate reader (BioTek, USA) using a wavelength of $570 \mathrm{~nm}$. Equation 2 was used to calculate cell viability. Triplicate samples were analyzed for each experiment [16]. IC50, the drug concentration at which inhibition of $50 \%$ cell growth was observed when compared to a control sample, was calculated from the appropriate curve of cell viability data.

$$
\text { Cell Viability }(\%)=\frac{\text { Absorbance of Sample }}{\text { Absorbance of Control }} \times 100 \quad \text { (Equation 2) }
$$




\subsubsection{Cellular uptake of 5-FU nano hydrogel.}

Rhodamine-123 entrapped 5-FU nano hydrogel was prepared to study the cellular uptake. This was prepared by the addition of a $40 \mu \mathrm{L}$ solution of $1 \mathrm{mg} / \mathrm{ml}$ concentration of Rhodamine-123 to $5 \mathrm{~mL}$ of prepared nano hydrogel dispersion under magnetic stirring for 4-5 $\mathrm{hr}$ in the dark and further centrifuged for $20 \mathrm{~min}$ at $20,000 \mathrm{rpm}$ to remove the unbound Rhodamine-123 [29]. For the cellular uptake study KB cells were grown at a density of $5 \times 10^{3}$ cells per well in 24 well plate at $37^{\circ} \mathrm{C}$ for $24 \mathrm{hr}$. After attachment of the cells, the media was removed and incubated with $100 \mu \mathrm{g} / \mathrm{ml}$ of Rhodamine-123 stained prepared nano hydrogel for $24 \mathrm{hr}$ and counterstained with Hoechst dye-containing media. The cells were then washed twice with PBS to remove the sample not taken up by the cells. Then, intracellular Rhodamine fluorescence in the cells was examined using an inverted fluorescent microscope (Carl Zeiss, Axiovert A1, Germany) at a magnification of 20X [14].

\subsubsection{Stability study.}

Stability study of the 5-FU nano hydrogel formulation was carried out to determine the effect of temperature on the stability of drug content and release over the defined period. The formulation was filled with a one $\mathrm{ml}$ glass prefilled syringe. This prefilled syringe was stored according to ICH Q1C stability testing for new dosage forms at accelerated condition $\left(40 \pm 2^{\circ} \mathrm{C}\right.$, $75 \pm 5 \% \mathrm{RH})$ and room temperature $\left(34 \pm 3^{\circ} \mathrm{C}, 47 \pm 5 \% \mathrm{RH}\right)$ for six month and refrigerated condition $\left(5^{\circ} \mathrm{C} \pm 3^{\circ} \mathrm{C}\right)$ for a period of twelve months [30]. Samples were withdrawn at predetermined intervals, and physical and chemical parameters were analyzed, i.e., appearance, viscosity, gelling temperature, and drug content. The viscosity and gelling temperature of the formulation were measured as per the predefined procedure. Drug release from the formulation was also performed at the end of the stability study to verify the release mechanism profile and sterility. Shelf life (time needed for $10 \%$ loss, t90) estimation of the drug in the formulations was determined from stability at refrigerated condition $\left(5^{\circ} \mathrm{C} \pm 3^{\circ} \mathrm{C}\right)$ for twelve months [31].

\section{Results and Discussion}

Characterizations such as gelling phenomenon, rheological property, morphology, stability, cytotoxicity, and cellular uptake study were performed and analyzed to ensure the properties of prepared 5-FU nano hydrogel for pharmaceutical application in drug delivery.

\subsection{FT-IR spectroscopy analysis.}

FT-IR spectra of MC, TSC salt, and dried nano hydrogel are presented in Figure 1. For MC, absorption peaks observed at $3469 \mathrm{~cm}^{-1}$ corresponds to the $\mathrm{O}-\mathrm{H}$ stretching vibration mode of the alcohol group. The peak at $2926 \mathrm{~cm}^{-1}$ and $2838 \mathrm{~cm}^{-1}$ is related to the C-H asymmetric and symmetric stretching, respectively. The absorption band at $1646 \mathrm{~cm}^{-1}$ represents the C-O carbonyl stretching in the anhydrous glucose unit of cellulose. The peak at $1120.64 \mathrm{~cm}^{-1}$ and $1058 \mathrm{~cm}^{-1}$ is related to the aliphatic ether C-O-C stretching. In the case of TSC, absorption peaks of $\mathrm{O}-\mathrm{H}$ stretching vibrations at $3270.79 \mathrm{~cm}^{-1}$ and $3452.30 \mathrm{~cm}^{-1}$, a medium stretching vibrations of $\mathrm{C}-\mathrm{H}$ bond at $2965.34 \mathrm{~cm}^{-1}$ and $2922.91 \mathrm{~cm}^{-1}$ (between 2964-2924 $\mathrm{cm}^{-1}$ ), and a strong asymmetric vibration of $\mathrm{C}=\mathrm{O}$ group at $1591.31 \mathrm{~cm}^{-1}$ (between $1695-1540 \mathrm{~cm}^{-1}$ ) which proves the existence of citrate as a salt [32]. When MC mixed with TSC, the peak at 1591.31 $\mathrm{cm}^{-1}$ due to the $\mathrm{C}=\mathrm{O}$ group was disappeared. The intensity of absorption bands of aliphatic 
ether at $1120.64 \mathrm{~cm}^{-1}$ and $1058 \mathrm{~cm}^{-1}$ diminish compared to pure $\mathrm{MC}$ that shows that there may be the formation of an intramolecular hydrogen bond between the hydroxyl group and methoxy group of MC. This mechanism appears very similar to those reported by Yuka Sekiguchi et al., in terms of the crosslinking and intramolecular hydrogen bond [22]. They have reported that in MC samples, methoxy groups are almost unable to form three-dimensional networks by themselves but are sterically able to form the intermolecular hydrogen bonds with the hydroxyl group, leading to crosslinking networks, which is identified by the absence of aliphatic ether peak. It proves that there is no direct chemical crosslinking between the TSC and MC components.

\subsection{Gelation time and gelling temperature.}

Accordingly, TTM, the thermoreversible gelling temperature of nano hydrogel solution, was found to be $37^{\circ} \mathrm{C}$ to $40^{\circ} \mathrm{C}$, and it forms a gel within 3 minutes. Further on cooling the same solution, it forms a solution at $\sim 20^{\circ} \mathrm{C}$. It shows the thermoreversible phenomenon of the prepared 5-FU nano hydrogel that is further confirmed by the rheological measurements.

\subsection{Rheological measurement.}

The viscosity of the 5-FU loaded thermosensitive nano hydrogel formulation at nonphysiological condition (solution state), and physiological condition (in gel state) was found to be $71.02 \pm 1.82 \mathrm{cP}$ and $2276 \pm 0.86 \mathrm{cP}$, respectively. The viscosity of the solution usually plays an important role in the flow properties of the material. To investigate the flow property of the formulation, viscosity measurement was made at an increasing shear rate, as shown in Figure 2. The flow behavior graph shows that the formulation exhibits a non-newtonian pseudoplastic flow [31]. The formulation did not collapse even at the higher shear rate of 1800, indicating good gel strength that is $66 \mathrm{gm}$. Gels strength was further confirmed by the time vs. load graph, as shown in Figure 3. Syringeability is the key product performance parameters for the parenteral dosage form. It refers to the ability of an injectable therapeutic to pass easily through a hypodermic needle on transfer from the injection. For a $24 \mathrm{G}$ needle, the force required for a 5-FU nano hydrogel solution was found to be $265 \mathrm{gm}$, and for WFI, it was found to be $149 \mathrm{gm}$. It was higher than WFI, which is because of the viscosity of nano hydrogel solution. 5-FU nano hydrogel solution shows the continuous flow from the $24 \mathrm{G}$ needle.

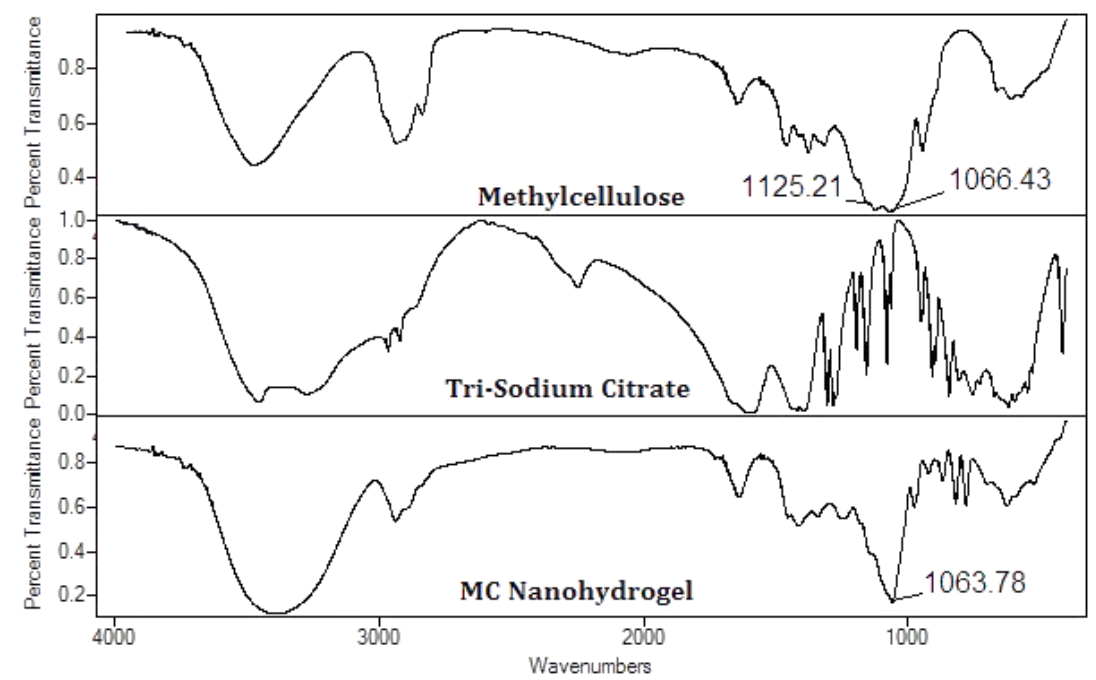

Figure 1. FT-IR spectra of methylcellulose, trisodium citrate, and MMC nano hydrogel. 


\subsection{Swelling study.}

The swelling study profile of 5-FU nano hydrogel in phosphate buffer $\mathrm{pH} 7.4$ are shown in figure 3 , and it was observed that that nano hydrogel could absorb water and saturated rapidly at $6 \mathrm{hr}$. The percentage swelling ratio of the nano hydrogel was found to be $122.66 \pm 1.52 \%$. Lower swelling and water uptake of the prepared nano hydrogel may be attributed to some $\mathrm{OH}^{-}$ group in the molecular chain. This $\mathrm{OH}^{-}$groups are involved in the $\mathrm{H}$-bond formation that ultimately results in lower water uptake of the gel [33].

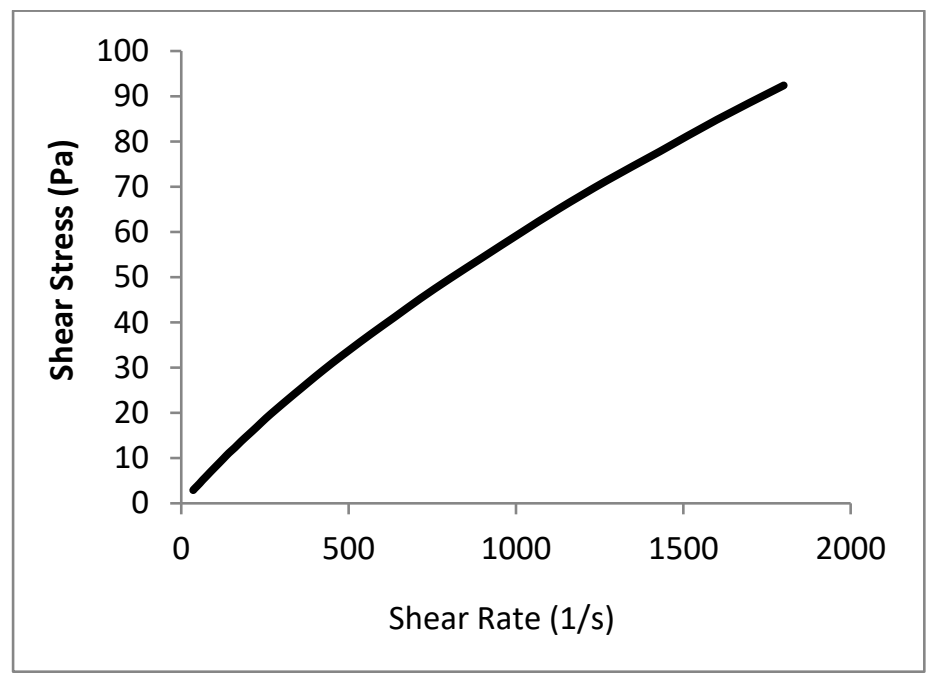

[A]

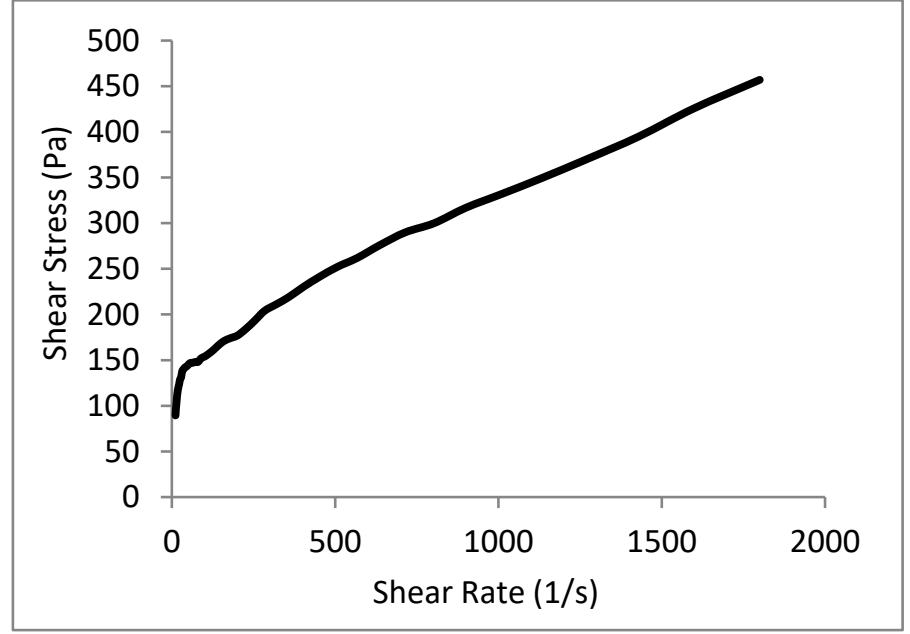

[B]

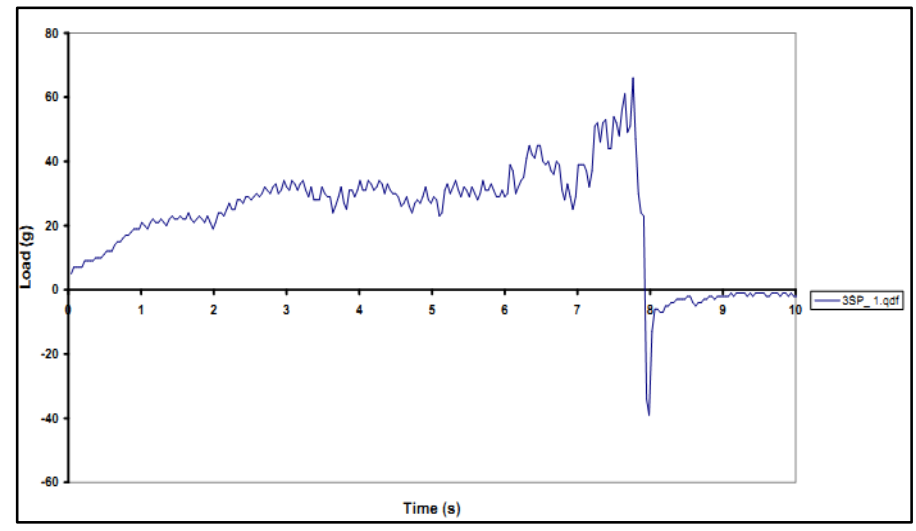

$[\mathrm{C}]$

Figure 2. Flow Curves for 5-FU nano hydrogel solution at non-physiological condition $[\mathrm{A}]$ and physiological condition [B] Time vs. load graph of gel strength for 5-FU nano hydrogel solution [C]. 


\subsection{In-vitro drug release.}

The cumulative percentage release of 5-FU from thermosensitive nano hydrogel and market formulation (Fluracil@, 500mg/10ml, Biochem Pharmaceutical Industries Limited, Mumbai, India) are shown in Figure 4. In contrast to the market formulation, it did not show burst release and gradually released the drug up to $24 \mathrm{hr}$. The different kinetic models show that the in-vitro drug release was best expressed by Higuchi's equation $\left(\mathrm{R}^{2}>0.9925\right)$ followed by first-order and zero-order kinetics. To confirm the diffusion mechanism, the data were fitted to the Korsmeyer-Peppas equation. It shows slope (n) value is 0.5 , indicating that diffusion is the predominant mechanism of drug release from these formulations.

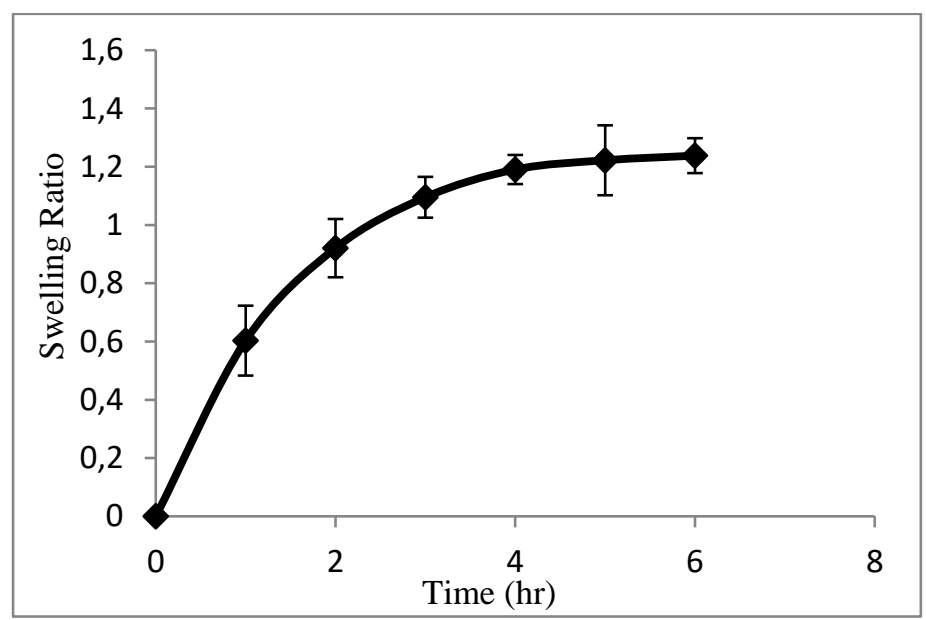

Figure 3. Swelling study profile of 5-FU nano hydrogel in phosphate buffer $\mathrm{pH} 7.4$ (mean $\mathrm{n}=3 \pm$ standard deviation).

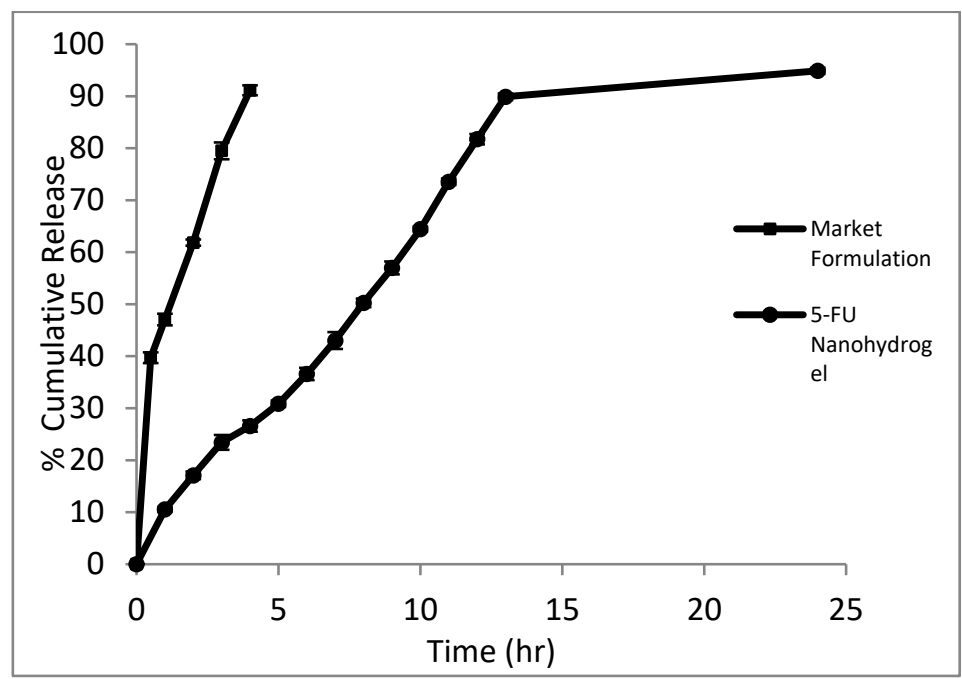

Figure 4. The drug release profile of 5-FU nano hydrogel, drug solution, and market formulation in phosphate buffer $\mathrm{pH} 7.4$ (mean $\mathrm{n}=3 \pm$ standard deviation).

\subsection{Morphology study.}

The morphology of the freeze-dried nano hydrogel is given in Figure 5. It displays the SEM images of the cross-section of the formulation. The nanohydrogels swollen at body physiological temperatures are freeze-dried before the SEM morphological investigation. It shows the uneven and interconnected porous structure, and the pore sizes are between 500 to $800 \mathrm{~nm}$. It shows the highly denser physical crosslinked structure. It shows that the freezedrying process could generate a honeycomb structure of the nano hydrogel and hydrogel. 


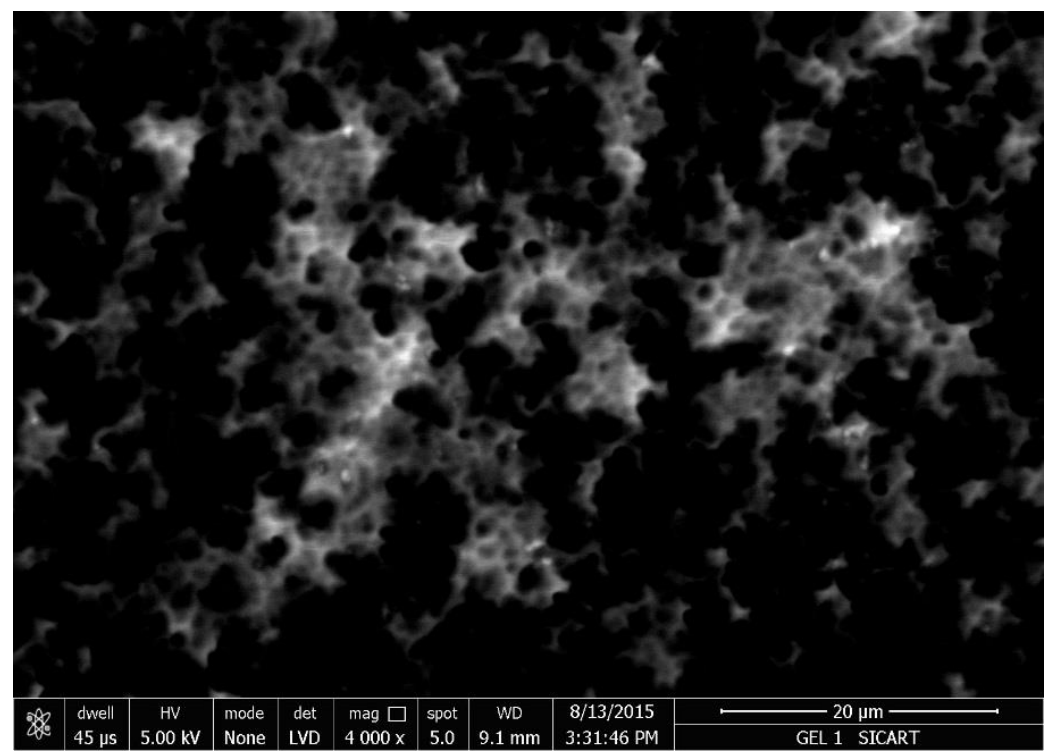

Figure 5. SEM images of the cross-section of freeze-dried nano hydrogel.

\subsection{Cytotoxicity assay and cellular uptake study.}

Cell toxicity was confirmed by the MTT colorimetric assay. Cell viability was measured after $24 \mathrm{hr}$ treatment with $\mathrm{KB}$ and VERO cells with the increasing concentration range of $1 \mu \mathrm{g} / \mathrm{ml}$ to $2000 \mu \mathrm{g} / \mathrm{ml}$ of $\mathrm{MC}$ polymer solution, drug solution, and 5-FU nanohydrogels.

In the case of $\mathrm{KB}$ oral cancer cells, both drug solution and drug-loaded nano hydrogel showed a similar concentration-dependent reduction in cell viability, as shown in Figure 6. The results demonstrated that the 5-FU could be well conserved in polymer and exerted the anticancer effect. At a concentration of $250 \mu \mathrm{g} / \mathrm{ml}$ both drug solution and nano hydrogel shows cell viability of $52.4 \%$ and $54.14 \%$, respectively. Also, blank polymer without 5-FU showed neglectable cytotoxicity with cell viability of 78-80\%. At lower concentrations, it did not have any effect on cell viability, but at a higher concentration, around $20 \%$ reduction in cell viability was observed [34].

Systemic study for cellular uptake of Rhodamine 123 conjugated 5-FU nano hydrogel by KB cells was performed by visualizing the fluorescence of Rhodamine 123 using a fluorescence microscope. Rhodamine 123 is a green fluorescence dye, so the cells with nano hydrogel uptake would typically appear bright green [29]. Figure 7A indicates the Rhodamine123 stained green fluorescence at the periphery of the cells, and Figure 7B shows the Hoechst dye stained nucleus of the cells, which gives blue color staining and further overlay of both images is observed in Figure $7 \mathrm{C}$ that clearly confirms the efficient uptake after $24 \mathrm{hr}$ incubation of the cells using Rhodamine-123 stained nano hydrogel with considerable green intracellular fluorescence in cells [14],[35].

Figure 8 depicts the toxicity of polymer and drug solution and 5-FU loaded nano hydrogel on the VERO fibroblast cell line. A non-linear decrease pattern in cell viability was observed for the prepared 5-FU nano hydrogel. The most intensive effect was observed at a higher concentration, at which approximately a $10 \%$ reduction in cell viability was produced compared to untreated control cells after $24 \mathrm{hr}$ exposures. Also, the drug solution in the same concentration range showed a $25-28 \%$ reduction in cell viability compared to 5-FU nano hydrogel [36]. 


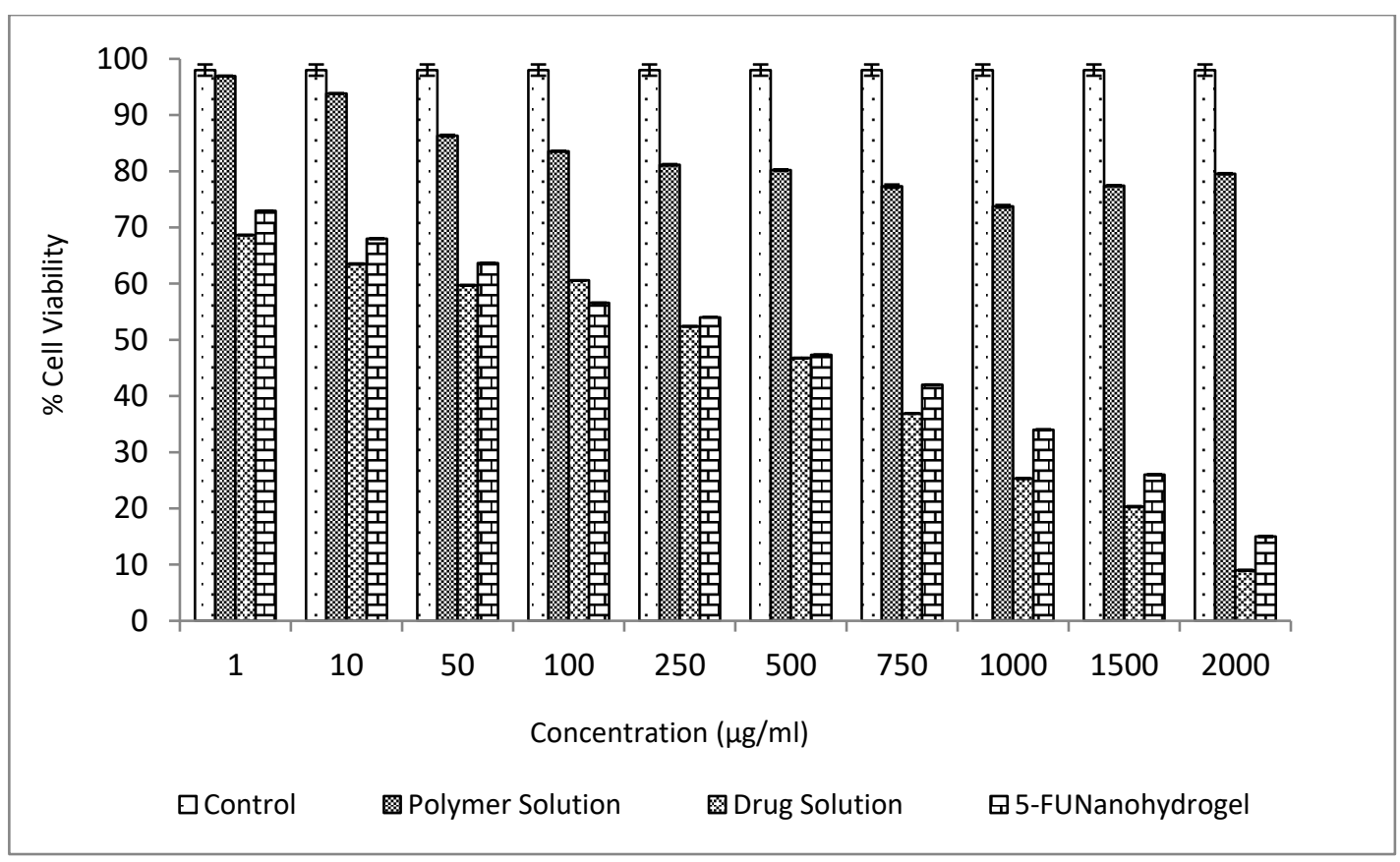

Figure 6. Cell viability of KB cells treated with 5-FU nano hydrogel, drug solution, and polymer solution after $24 \mathrm{hr}$ (mean $\mathrm{n}=3 \pm$ standard deviation).

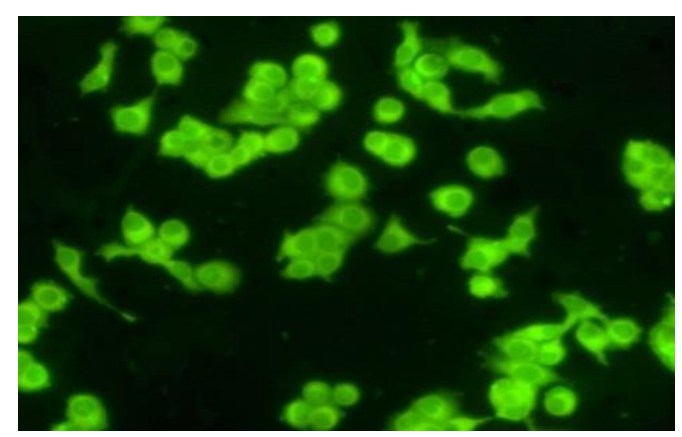

$[\mathrm{A}]$

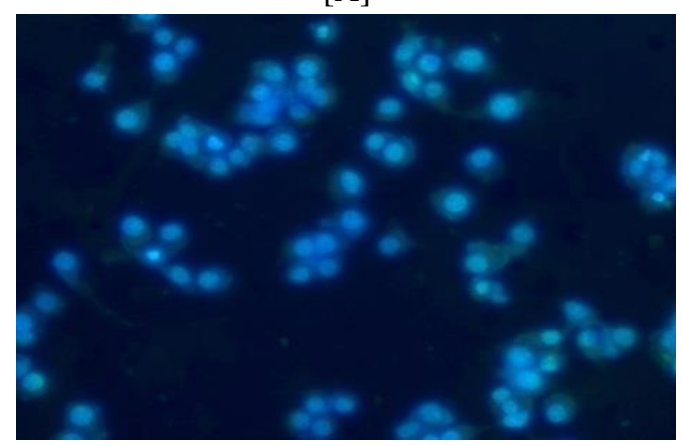

[B]

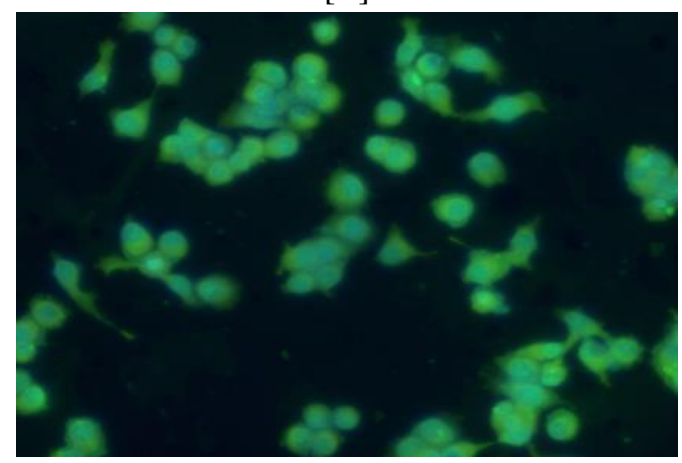

$[\mathrm{C}]$

Figure 7. Rhodamine-123 Stained [A] Hoechst stained [B] Overlay [C] fluorescent microphotographs obtained after $24 \mathrm{hr}$ incubation of KB cells with 5-FU nano hydrogel. 


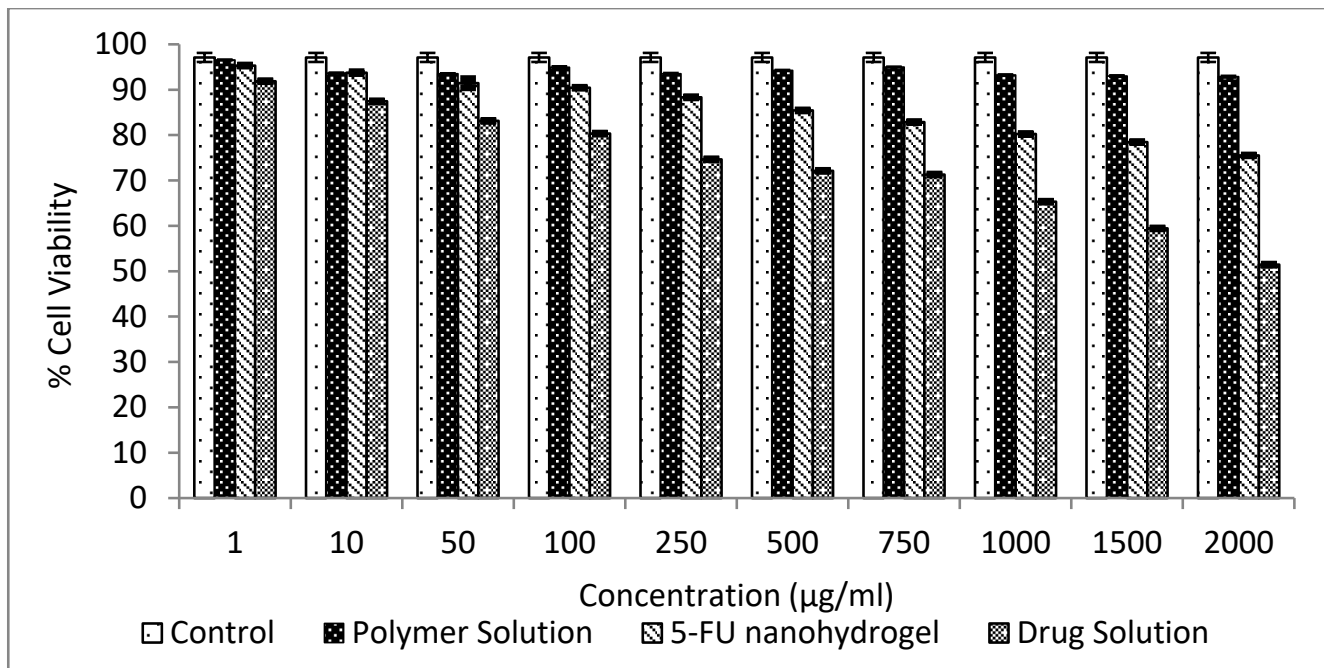

Figure 8. Cell viability of VERO cells treated with a drug solution, polymer solution, and 5-FU nano hydrogel after $24 \mathrm{hr}$ (mean $\mathrm{n}=3 \pm$ standard deviation).

\subsection{Stability study.}

The purpose of this study was to establish the recommended storage conditions. At accelerated conditions $\left(40^{\circ} \mathrm{C} \pm 2^{\circ} \mathrm{C}, 75 \% \pm 5 \% \mathrm{RH}\right)$ and room temperature $\left(34 \pm 3^{\circ} \mathrm{C}, 47 \pm 5 \%\right.$ $\mathrm{RH})$, drug-loaded nano hydrogel forms a gel and viscous solution respectively over the period of time. The formulation did not show any changes in physical and chemical properties at the refrigerated condition for a period of 12 months. The results of the stability study are given in Table 1 and Table 2. After refrigerated and accelerated conditions, 5-FU content was found to be $>95 \%$. Similar findings were observed by Tarek Ahmed et al. [37]. who studied the stability of haloperidol in in-situ implant and microparticle injectable formulation over a period of 12 months. The drug release profile at the end of the refrigerated stability condition obtained is given in Figure 9. The $\mathrm{f} 2$ value from the drug release profile for the first day and after 12 months was calculated and found 67.45. It revealed that there was no significant change observed in the release profile data. From the results, it was found that refrigerated condition was a desirable storage condition for the prepared 5-FU nano hydrogel. The proposed retest period or shelf life estimation of the final formulation for the refrigerated condition was found to be 1.5 years.

Table 1. Stability parameters at room temperature and accelerated condition of 5-FU nano hydrogel (mean $n=3$

\begin{tabular}{|c|c|c|c|c|c|c|c|}
\hline \multirow[t]{2}{*}{$\begin{array}{l}\text { Time } \\
\text { (Month) }\end{array}$} & \multicolumn{3}{|c|}{$\begin{array}{c}\text { Accelerated Condition } \\
\left(40^{\circ} \mathrm{C} \pm 2^{\circ} \mathrm{C}, \mathbf{7 5} \% \pm 5 \% \mathrm{RH}\right)\end{array}$} & \multicolumn{4}{|c|}{ Room Temperature $\left(34 \pm 3^{\circ} \mathrm{C}, 47 \pm 5 \% \mathrm{RH}\right)$} \\
\hline & Appearance & $\begin{array}{c}\text { Gelling } \\
\text { Temperature } \\
\left({ }^{\circ} \mathrm{C}\right)\end{array}$ & $\begin{array}{l}\% \text { Drug } \\
\text { Content }\end{array}$ & Appearance & $\begin{array}{c}\text { Gel } \\
\text { Temperature } \\
\left({ }^{\circ} \mathrm{C}\right)\end{array}$ & $\begin{array}{l}\% \text { Drug } \\
\text { Content }\end{array}$ & $\begin{array}{l}\text { Viscosity } \\
\quad(\mathrm{cP})\end{array}$ \\
\hline 0 & $\begin{array}{l}\text { Liquid (Then } \\
\text { forms gel) }\end{array}$ & $39 \pm 1.56$ & $99.8 \pm 0.17$ & $\begin{array}{l}\text { Liquid (Then } \\
\text { forms gel) }\end{array}$ & $39 \pm 2$ & $99.7 \pm 0.17$ & $70.1 \pm 0.08$ \\
\hline 0.5 & Gel & $39 \pm 1.72$ & $99.5 \pm 0.24$ & $\begin{array}{c}\text { Viscous } \\
\text { liquid } \\
\end{array}$ & $39 \pm 1.66$ & $99.1 \pm 0.11$ & $70.6 \pm 0.12$ \\
\hline 1 & Gel & $39 \pm 1.93$ & $99.6 \pm 0.26$ & $\begin{array}{l}\text { Viscous } \\
\text { liquid } \\
\end{array}$ & $39 \pm 1.77$ & $99.6 \pm 0.07$ & $71.5 \pm 0.02$ \\
\hline 2 & Gel & $39 \pm 1.02$ & $99.3 \pm 0.09$ & $\begin{array}{l}\text { Viscous } \\
\text { liquid }\end{array}$ & $39 \pm 1.41$ & $99.4 \pm 0.15$ & $75.2 \pm 0.21$ \\
\hline 3 & Gel & $39 \pm 1.88$ & $99.7 \pm 0.11$ & $\begin{array}{l}\text { Viscous } \\
\text { liquid }\end{array}$ & $39 \pm 1.39$ & $99.7 \pm 0.16$ & $76.9 \pm 0.18$ \\
\hline 6 & Gel & $39 \pm 1.53$ & $99.0 \pm 0.16$ & $\begin{array}{l}\text { Viscous } \\
\text { liquid }\end{array}$ & $39 \pm 1.9$ & $99.5 \pm 0.03$ & $82.1 \pm 0.15$ \\
\hline
\end{tabular}


Table 2. Stability parameters at the refrigerated condition of 5-FU nano hydrogel (mean $n=3 \pm$ standard deviation).

\begin{tabular}{c|c|c|c|c}
\multirow{2}{*}{$\begin{array}{c}\text { Time } \\
\text { Months) }\end{array}$} & \multicolumn{4}{|c}{ Refrigerated Condition $\left(\mathbf{5}^{\circ} \mathbf{C} \pm \mathbf{3}^{\circ} \mathbf{C}\right)$} \\
\cline { 2 - 5 } & Appearance & Gel temperature $\left({ }^{\circ} \mathrm{C}\right)$ & $\%$ Drug Content & Viscosity $(\mathrm{cP})$ \\
\hline 0 & Liquid & $39 \pm 1.56$ & $99.70 \pm 0.07$ & $70.1 \pm 0.08$ \\
\hline 1 & Liquid & $39 \pm 1.61$ & $99.42 \pm 0.14$ & $68.8 \pm 0.18$ \\
\hline 2 & Liquid & $39 \pm 1.85$ & $99.52 \pm 0.16$ & $68.3 \pm 0.07$ \\
\hline 3 & Liquid & $39 \pm 1.44$ & $99.21 \pm 0.19$ & $69.6 \pm 0.11$ \\
\hline 6 & Liquid & $39 \pm 1.79$ & $99.63 \pm 0.21$ & $69.8 \pm 0.13$ \\
\hline 9 & Liquid & $39 \pm 1.15$ & $99.17 \pm 0.1$ & $69.2 \pm 0.07$ \\
\hline 12 & Liquid & $39 \pm 1.28$ & $99.01 \pm 0.25$ & $69.0 \pm 0.21$
\end{tabular}

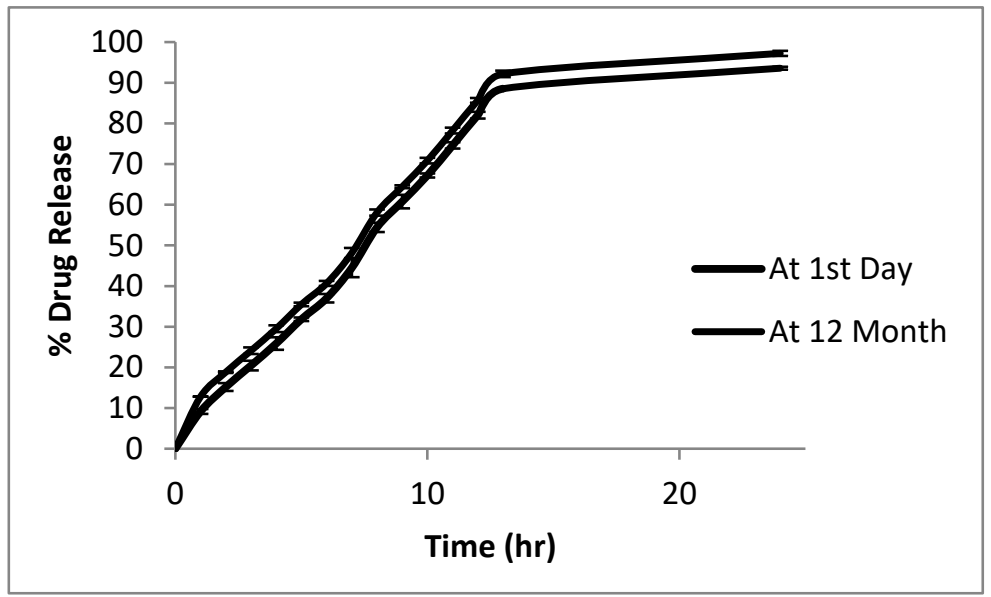

Figure 9. Drug release profile comparison at refrigerated stability condition (mean $n=3 \pm$ standard deviation).

\section{Conclusions}

Here in this study, thermoresponsive 5-FU nano hydrogel with a pore size of $500 \mathrm{~nm}$ to $800 \mathrm{~nm}$ was prepared from MMC hydrogel by probe sonication method and characterized for cytotoxicity and cellular uptake study. A rheological investigation of the swollen nanohydrogels confirmed that it follows pseudoplastic flow behavior. Swelling experiments indicated that the nanohydrogels shows water uptake with fast swelling/deswelling responses and undergo oscillatory swelling in response to changes in the temperature. Cytotoxicity assay using $\mathrm{KB}$ oral cancer cell line indicates that the drug efficacy toward cancer cells remained unchanged after incorporation in the nano hydrogel and also shows efficient cellular uptake. In addition to the VERO fibroblast cells, the 5-FU nano hydrogel shows negligible toxicity compared to the drug solution that also proves the biocompatibility of the formulation. Although these results are desirable, an in-vivo pharmacokinetic and pharmacodynamic study is further required to prove intratumor targeting as a localized promising approach.

\section{Funding}

This research received no external funding.

\section{Acknowledgments}

The authors are thankful to Principal Ramanbhai Patel College of Pharmacy, Charotar University of Science and Technology (CHARUSAT), and K. C. Patel Research and Development Centre (KRADLE) for providing facilities for this research work. 


\section{Conflicts of Interest}

\section{The authors declare no conflict of interest.}

\section{References}

1. Pfister, D.; Spencer, S.; Brizel, D.; Burtness, B.; Busse, P.; Caudell, J.; Cmelak, A.; Colevas, A.; Dunphy, F.; Eisele, D.; Foote, R.; Gilbert, J.; Gillison, M.; Haddad, R.; Haughey, B.; Hicks, W.; Hitchcock, Y.; Jimeno, A.; Kies, M.; Hughes, M. Head and Neck Cancers, Version 1.2015 Featured Updates to the NCCN Guidelines. JNCCN Journal of the National Comprehensive Cancer Network 2015, 13, 847-856, https://doi.org/10.6004/jnccn.2013.0113.

2. Head and Neck Cancer; Union for International Cancer Control. 2014 Review of Cancer Medicines on the WHO List of Essential Medicines. Locally Advanced Squamous Carcinoma of The Head And Neck.

3. Joshi, P.; Dutta, S.; Chaturvedi, P.; Nair, S. Head and neck cancers in developing countries. Rambam Maimonides Med J 2014, 5, 1-6, https://doi.org/10.5041/RMMJ.10143.

4. Mishra, A.; Meherotra, R. Head and neck cancer: global burden and regional trends in India. Asian Pacific journal of cancer prevention : APJCP 2014, 15, 537-550, https://doi.org/10.7314/apjcp.2014.15.2.537.

5. Kulkarni, M. Head and Neck Cancer Burden in India. International Journal of Head and Neck Surgery 2013, 4, 29-35.

6. Kenneth, H.J.W. The management of head and neck cancer. Surgery 2009, 27, 540-545.

7. Bhide, S.A.; Nutting, C.M. Advances in chemotherapy for head and neck cancer. Oral Oncology 2010, 46, 436-438, https://doi.org/10.1016/j.oraloncology.2010.03.004.

8. World health Organization Classification of Tumors. Pathology and Genetics of Head and Neck Tumors. International Agency for Research on Cancer: Lyon, France, 2005.

9. Wolinsky, J.B.; Colson, Y.L.; Grinstaff, M.W. Local drug delivery strategies for cancer treatment: gels, nanoparticles, polymeric films, rods, and wafers. $J$ Control Release 2012, 159, 14-26, https://doi.org/10.1016/j.jconrel.2011.11.031.

10. National Cancer Institute. Fact sheet: Head and Neck Cancer. 2012.

11. Abouelfadel, Z.; Crawford, E.D. Leuprorelin depot injection: patient considerations in the management of prostatic cancer. Ther Clin Risk Manag 2008, 4, 513-526, https://doi.org/10.2147/tcrm.s6863.

12. Dalwadi, C.; Patel, G. Application of nanohydrogels in drug delivery systems: recent patents review. Recent patents on nanotechnology 2015, 9, 17-25, https://doi.org/10.2174/1872210509666150101151521.

13. Zhu, L.; Ma, J.; Jia, N.; Zhao, Y.; Shen, H. Chitosan-coated magnetic nanoparticles as carriers of 5fluorouracil: preparation, characterization and cytotoxicity studies. Colloids Surf B Biointerfaces 2009, 68, 1-6, https://doi.org/10.1016/j.colsurfb.2008.07.020.

14. Nair K, L.; Jagadeeshan, S.; Nair, S.A.; Kumar, G.S.V. Biological evaluation of 5-fluorouracil nanoparticles for cancer chemotherapy and its dependence on the carrier, PLGA. International journal of nanomedicine 2011, 6, 1685-1697, https://doi.org/10.2147/IJN.S20165.

15. Rejinold, N.S.; Chennazhi, K.P.; Nair, S.V.; Tamura, H.; Jayakumar, R. Biodegradable and thermo-sensitive chitosan-g-poly(N-vinylcaprolactam) nanoparticles as a 5-fluorouracil carrier. Carbohydrate Polymers 2011, 83, 776-786, https://doi.org/10.1016/j.carbpol.2010.08.052.

16. Kevadiya, B.D.; Patel, T.A.; Jhala, D.D.; Thumbar, R.P.; Brahmbhatt, H.; Pandya, M.P.; Rajkumar, S.; Jena, P.K.; Joshi, G.V.; Gadhia, P.K.; Tripathi, C.B.; Bajaj, H.C. Layered inorganic nanocomposites: A promising carrier for 5-fluorouracil (5-FU). European Journal of Pharmaceutics and Biopharmaceutics 2012, 81, 91101, https://doi.org/10.1016/j.ejpb.2012.01.004.

17. Ta, H.T.; Dass, C.R.; Dunstan, D.E. Injectable chitosan hydrogels for localised cancer therapy. J Control Release 2008, 126, 205-216, https://doi.org/10.1016/j.jconrel.2007.11.018.

18. Guerrero-Ramírez, L.G.; Nuño-Donlucas, S.M.; Cesteros, L.C.; Katime, I. Smart copolymeric nanohydrogels: Synthesis, characterization and properties. Materials Chemistry and Physics 2008, 112, 1088-1092, https://doi.org/10.1016/j.matchemphys.2008.07.023.

19. Chacko, R.T.; Ventura, J.; Zhuang, J.; Thayumanavan, S. Polymer nanogels: A versatile nanoscopic drug delivery platform. Advanced Drug Delivery Reviews 2012, 64, 836-851, https://doi.org/10.1016/j.addr.2012.02.002.

20. Zahedi, P.; Stewart, J.; De Souza, R.; Piquette-Miller, M.; Allen, C. An injectable depot system for sustained intraperitoneal chemotherapy of ovarian cancer results in favorable drug distribution at the whole body, peritoneal and intratumoral levels. Journal of Controlled Release 2012, 158, 379-385, https://doi.org/10.1016/j.jconrel.2011.11.025.

21. D'Arrigo, G.; Navarro, G.; Di Meo, C.; Matricardi, P.; Torchilin, V. Gellan gum nanohydrogel containing anti-inflammatory and anti-cancer drugs: a multi-drug delivery system for a combination therapy in cancer treatment. European journal of pharmaceutics and biopharmaceutics : official journal of Arbeitsgemeinschaft fur Pharmazeutische Verfahrenstechnik e.V 2014, 87, 208-216, https://doi.org/10.1016/j.ejpb.2013.11.001. 
22. Sekiguchi, Y.; Sawatari, C.; Kondo, T. A gelation mechanism depending on hydrogen bond formation in regioselectively substituted O-methylcelluloses. Carbohydrate Polymers 2003, 53, 145-153, https://doi.org/10.1016/S0144-8617(03)00050-X.

23. Fathi, M.; Entezami, A.A.; Pashaei-Asl, R. Swelling/deswelling, thermal, and rheological behavior of PVAg-NIPAAm nanohydrogels prepared by a facile free-radical polymerization method. Journal of Polymer Research 2013, 20, 125-136, https://doi.org/10.1007/s10965-013-0125-5.

24. Liu, K.-H.; Liu, T.-Y.; Chen, S.-Y.; Liu, D.-M. Drug release behavior of chitosan-montmorillonite nanocomposite hydrogels following electrostimulation. Acta Biomaterialia 2008, 4, 1038-1045, https://doi.org/10.1016/j.actbio.2008.01.012.

25. Wang, Y.; Xu, H.; Wang, J.; Ge, L.; Zhu, J. Development of a Thermally Responsive Nanogel Based on Chitosan-Poly(N-Isopropylacrylamide-co-Acrylamide) for Paclitaxel Delivery. Journal of Pharmaceutical Sciences 2014, 103, 2012-2021, https://doi.org/10.1002/jps.23995.

26. Li, X.; Li, R.; Qian, X.; Ding, Y.; Tu, Y.; Guo, R.; Hu, Y.; Jiang, X.; Guo, W.; Liu, B. Superior antitumor efficiency of cisplatin-loaded nanoparticles by intratumoral delivery with decreased tumor metabolism rate. European Journal of Pharmaceutics and Biopharmaceutics 2008, 70, 726-734, https://doi.org/10.1016/j.ejpb.2008.06.016.

27. Zhang, J.; Qian, Z.; Gu, Y. In vivo anti-tumor efficacy of docetaxel-loaded thermally responsive nanohydrogel. Nanotechnology 2009, 20.

28. Xia, L.-W.; Xie, R.; Ju, X.-J.; Wang, W.; Chen, Q.; Chu, L.-Y. Nano-structured smart hydrogels with rapid response and high elasticity. Nature Communications 2013, 4, 1-11, https://doi.org/10.1038/ncomms3226.

29. Sabitha, M.; Sanoj Rejinold, N.; Nair, A.; Lakshmanan, V.-K.; Nair, S.V.; Jayakumar, R. Development and evaluation of 5-fluorouracil loaded chitin nanogels for treatment of skin cancer. Carbohydrate Polymers 2013, 91, 48-57, https://doi.org/10.1016/j.carbpol.2012.07.060.

30. International Conference on Harmonization of Technical Requirements for Registration of Pharmaceuticals for Human Use (ICH): Stability Testing for New Dosage Forms Q1C, 1996.

31. Fathi, M.; Reza Farajollahi, A.; Akbar Entezami, A. Synthesis of fast response crosslinked PVA-g-NIPAAm nanohydrogels by very low radiation dose in dilute aqueous solution. Radiation Physics and Chemistry 2013, 86, 145-154, https://doi.org/10.1016/j.radphyschem.2013.02.005.

32. Demitri, C.; Del Sole, R.; Scalera, F.; Sannino, A.; Vasapollo, G.; Maffezzoli, A.; Ambrosio, L.; Nicolais, L. Novel superabsorbent cellulose-based hydrogels crosslinked with citric acid. Journal of Applied Polymer Science 2008, 110, 2453-2460, https://doi.org/10.1002/app.28660.

33. Nair, S.; Remya, N.S.; Remya, S.; Nair, P.D. A biodegradable in situ injectable hydrogel based on chitosan and oxidized hyaluronic acid for tissue engineering applications. Carbohydrate Polymers 2011, 85, 838-844, https://doi.org/10.1016/j.carbpol.2011.04.004.

34. Zhang, J.; Chen, H.; Xu, L.; Gu, Y. The targeted behavior of thermally responsive nanohydrogel evaluated by NIR system in mouse model. Journal of Controlled Release 2008, 131, 34-40, https://doi.org/10.1016/j.jconrel.2008.07.019.

35. Rejinold, N.S.; Muthunarayanan, M. 5-Fluorouracil loaded fibrinogen nanoparticles for cancer drug delivery applications. International Journal of Biological Macromolecules 2011, 48, 98-105.

36. Rejinold, N.S.; Nair, A.; Sabitha, M.; Chennazhi, K.P.; Tamura, H.; Nair, S.V.; Jayakumar, R. Synthesis, characterization and in vitro cytocompatibility studies of chitin nanogels for biomedical applications. Carbohydrate Polymers 2012, 87, 943-949, https://doi.org/10.1016/j.carbpol.2011.08.044.

37. Ahmed, T.A.; Ibrahim, H.M.; Ibrahim, F.; Samy, A.M.; Kaseem, A.; H. Nutan, M.T.; Hussain, M.D. Development of Biodegradable In Situ Implant and Microparticle Injectable Formulations for Sustained Delivery of Haloperidol. Journal of Pharmaceutical Science 2012, 101, 3753-3762, https://doi.org/10.1002/jps.23250. 